\title{
Plasma Leptin Concentration in Dogs with Diabetes Mellitus
}

\author{
Naohito NISHII ${ }^{1) *}$, Miho YAMASAKI ${ }^{2}$, Masaki TAKASU ${ }^{3)}$, Tsutomu HONJOH ${ }^{4}$, Haruki SHIBATA ${ }^{4)}$, \\ Yoshihiko OTSUKA ${ }^{5)}$, Satoshi TAKASHIMA ${ }^{2)}$, Yasunori $\mathrm{OHBA}^{2)}$ and Hitoshi KITAGAWA ${ }^{2)}$ \\ ${ }^{1)}$ Laboratory of Veterinary Pharmacotherapeutics, Faculty of Agriculture, Tottori University, 4-101 Minami, Koyama-cho, Tottori 680-5883, \\ ${ }^{2)}$ Laboratory of Veterinary Internal Medicine, Faculty of Applied Biological Sciences, Gifu University, 1-1 Yanagido, Gifu 501-1193, \\ ${ }^{3)}$ Laboratory of Veterinary Clinical Theriogenology, Faculty of Applied Biological Sciences, Gifu University, 1-1 Yanagido, Gifu 501- \\ 1193, 4) Morinaga Institute of Biological Science, Inc., 2-1-16 Sachiura, Kanazawa-ku, Yokohama, Kanagawa 236-0003 and \\ ${ }^{5)}$ Mitsubishi Chemical Medience Corporation, 30-1-3 Shimura, Itabashi-ku, Tokyo 174-8555, Japan
}

(Received 26 October 2009/Accepted 28 January 2010/Published online in J-STAGE 9 February 2010)

ABSTRACT. The plasma leptin concentration was evaluated in dogs with diabetes mellitus. Twenty normal and sixteen diabetic dogs were divided into nonobese and obese groups based on body condition score, respectively. The obese normal dogs had significantly higher plasma leptin concentrations than the nonobese normal dogs, whereas there was no significant difference between the nonobese and obese diabetic dogs. In addition, the plasma leptin concentration in the obese diabetic dogs was significantly lower than that in the obese normal dogs. In conclusion, the plasma leptin concentrations in the diabetic dogs were affected by factors other than adiposity. KEY WORDS: canine, diabetes, insulin, leptin.

Leptin is a product of the $o b$ gene, which is mainly secreted from adipose tissue [16]. It regulates body weight through modulation of feeding behavior and energy expenditure [2]. In addition, leptin is known to have an important role in glucose and lipid metabolism [2]. It is also known to have positive correlation to adiposity in humans [3] and dogs [7]. Other than adiposity, insulin is one of factors that regulate the plasma leptin concentration, and it has been shown that an insulin administration increases the plasma leptin concentration [8]. Insulin administration has also been shown to increase the plasma leptin concentration in dogs [6]. Since leptin has an intimate involvement in insulin secretion or insulin resistance [16], it can contribute to the pathophysiology of diabetes mellitus. However, plasma leptin concentrations have not been investigated in dogs with diabetes. In the present study, we determined plasma leptin concentrations in diabetic dogs.

This study included 36 dogs brought to the Veterinary Teaching Hospital of Gifu University (Table 1). Sixteen of them were dogs newly diagnosed with diabetes mellitus based on clinical signs, physical examination, continuously high plasma glucose concentrations, and detection of glucosuria. All diabetic dogs required insulin treatment, and none of them were transient hyperglycemia, such as diestrus- and gestation-associated diabetes. The remaining twenty were age- and body condition-matched normal dogs diagnosed as clinically healthy based on physical examination, complete blood count and biochemical analysis. The diabetic and normal dogs were divided into two groups, the nonobese and obese groups, based on body condition score (BCS) in accordance with a 9-point scale [9] (BCSs of 4 to 6 and 7 to

\footnotetext{
* Correspondence to: Nishin, N., Laboratory of Veterinary Pharmacotherapeutics, Faculty of Agriculture, Tottori University, 4101 Minami, Koyama-cho, Tottori 680-5883, Japan. e-mail: nnishii@muses.tottori-u.ac.jp
}

9 were considered nonobese and obese, respectively). This study was conducted in a manner consistent with the Gifu University Guidelines for Animal Experimentation. Fasting venous blood samples were collected into heparin-coated tubes and then centrifuged. Isolated plasma samples were stored at $-30^{\circ} \mathrm{C}$ until measurement of the plasma leptin and insulin concentrations. The plasma leptin and insulin concentrations were determined by the same methods, an enzyme-linked immunosorbent assay and an immunoradiometric assay, respectively, as used in our previous study [10]. Differences in variables between the groups were determined by the Mann-Whitney test. Correlation between the plasma leptin concentration and the variables was evaluated by use of the Spearman rank correlation coefficient. Probabilities of less than 0.05 were considered to be statistically significant.

The plasma leptin concentrations of the normal and diabetic dogs are shown in Fig. 1. The obese normal dogs had significantly higher plasma leptin concentration than the nonobese normal dogs $(13.1 \pm 7.6$ vs $1.1 \pm 0.3 \mathrm{ng} / \mathrm{m} l)$. On the other hand, there was no significant difference between the obese diabetic and the nonobese diabetic dogs (1.5 \pm 1.0 vs $2.3 \pm 2.7 n \mathrm{~g} / \mathrm{m} l)$. No difference was observed between the nonobese diabetic and the nonobese normal dogs. The plasma leptin concentrations of the obese diabetic dogs were significantly lower than those of the obese normal dogs. The plasma leptin concentration was significantly correlated with age and BCS in the normal dogs, whereas it was not in the diabetic dogs (Table 2). Other variables, including plasma glucose, insulin, cholesterol and triglyceride concentrations, showed no correlation with the plasma leptin concentration in both the normal and diabetic dogs.

To our knowledge, this is the first study reporting about the plasma leptin concentration in dogs with diabetes mellitus. The results of the present study showed that there was 
Table 1. Characteristics and plasma glucose and insulin concentrations in normal and diabetic dogs divided into nonobese and obese groups

\begin{tabular}{lccccc}
\hline & \multicolumn{2}{c}{ Normal } & & \multicolumn{2}{c}{ Diabetic } \\
\cline { 2 - 3 } \cline { 5 - 6 } Variable & Nonobese $(\mathrm{n}=10)$ & Obese $(\mathrm{n}=10)$ & & Nonobese $(\mathrm{n}=11)$ & Obese $(\mathrm{n}=5)$ \\
\hline Sex $(\mathrm{male} / \mathrm{female})$ & $5 / 5$ & $3 / 7$ & & $3 / 8$ & $1 / 4$ \\
Age (years) & $7.4 \pm 1.8$ & $9.7 \pm 1.8$ & & $9.0 \pm 2.3$ & $9.8 \pm 2.2$ \\
BCS & $5.3 \pm 0.5$ & $7.0 \pm 0$ & & $5.2 \pm 0.8$ & $7.2 \pm 0.4$ \\
Glucose $(\mathrm{mg} / \mathrm{d} l)$ & $95.7 \pm 11.6$ & $96.1 \pm 14.4$ & & $446.9 \pm 110.3^{*}$ & $569.4 \pm 371.9^{*}$ \\
Insulin $(n \mathrm{~g} / \mathrm{m} l)$ & $0.5 \pm 0.5$ & $0.9 \pm 0.9$ & & $0.6 \pm 0.9$ & $1.2 \pm 1.5$ \\
Cholesterol $(\mathrm{mg} / \mathrm{d} l)$ & $201.1 \pm 70.8$ & $293.1 \pm 96.6^{\dagger}$ & & $310.6 \pm 90.5 *$ & $255.8 \pm 78.5$ \\
Triglyceride $(\mathrm{mg} / \mathrm{d} l)$ & $73.3 \pm 41.1$ & $131.7 \pm 112.5$ & & $190.8 \pm 173.7^{*}$ & $134.0 \pm 66.7$ \\
\hline
\end{tabular}

Data are expressed as means $\pm \mathrm{SD}$.

* Significantly $(P<0.05)$ different from the values of the BCS-matched normal dogs.

${ }^{\dagger}$ Significantly $(P<0.05)$ different from the values of the nonobese normal dogs.

BCS: Body condition score in 9-point scale.



Fig. 1. The plasma leptin concentrations of the normal and diabetic dogs divided into the nonobese and obese groups. * Significantly $(P<0.05)$ different from the values of the nonobese normal dogs. $\dagger$ Significantly $(P<0.05)$ different from the values of the obese normal dogs.

significantly lower concentration of leptin in the obese diabetic dogs compared with the obese normal dogs. The low plasma leptin concentration in diabetic patients is consistent with the previous results for human type $1[5,11]$ and 2 diabetes $[1,11,13]$. The plasma leptin concentration showed significant correlation with age and BCS in the normal dogs, as previously known [7], however, there was no such correlation in the diabetic dogs. In other words, plasma leptin concentrations did not increase along with age or BCS in the diabetic dogs. Although age and BCS are important determinants for the plasma leptin concentration, other might largely affect the plasma leptin concentration in diabetic dogs. One of possible causes for the disturbance of leptin concentration in diabetic dogs is insulin deficiency. Because insulin administration increases the blood leptin
Table 2. Correlation analysis between the plasma leptin concentration and the variables in the normal and diabetic dogs

\begin{tabular}{lccrrr}
\hline \multirow{2}{*}{ Variable } & \multicolumn{2}{c}{ Normal $(\mathrm{n}=20)$} & & \multicolumn{2}{c}{ Diabetic $(\mathrm{n}=16)$} \\
\cline { 2 - 3 } \cline { 5 - 6 } & $r s$ & $P$ & & $r s$ & $P$ \\
\hline Age & 0.61 & $<0.001$ & & 0.13 & NS \\
BCS & 0.86 & $<0.001$ & & 0.17 & NS \\
Glucose & 0.32 & NS & & -0.03 & NS \\
Insulin & 0.35 & NS & & 0.37 & NS \\
Cholesterol & 0.42 & NS & & 0.19 & NS \\
Triglyceride & 0.30 & NS & & -0.53 & NS \\
\hline
\end{tabular}

$r s$ : Rank correlation coefficient. $P$ : Probability.

BCS: Body condition score in 9-point scale.

NS: Not significant

concentration $[6,8]$, deficiency of insulin effect can result in a low blood leptin concentration. However, the plasma insulin concentration in the diabetic dogs showed no correlation with the plasma leptin concentration, was not significantly different from that in the normal dogs. This might be because the dogs in the present study were sampled under fasting conditions, or because some of the diabetic dogs had high fasting plasma insulin concentrations, which indicate existence of insulin resistance. Canine diabetes mellitus has been believed to be a disease caused by absolute deficiency of insulin secretion or relative deficiency of insulin by other pathology such as insulin resistance [4]. The limitation of the present study is the heterogenous pathologic type of diabetes mellitus. The lack of detailed pathologic data complicates further discussion. However, in both mechanisms, absolute and relative deficiency of insulin, the plasma leptin concentration in dogs with diabetes possibly becomes lower than in normal dogs.

On the other hand, some studies in human diabetes have shown that the blood leptin concentration is high [15] or unchanged $[12,14]$. However, the patients included in some of these studies had received treatments such as insulin administration. Since insulin administration positively affects the blood leptin concentration $[6,8]$, the discrepancy in the leptin concentration among the previous studies might be due to the difference in experience of the treatments. 
Indeed, insulin treatment significantly increases the leptin concentration in patients with type 1 diabetes [5].

Polyphagia is one of symptoms of canine diabetes mellitus. Because leptin acts to suppress appetite, deficiency of leptin can cause an increase of appetite [2]. The present results suggest that polyphagia observed in dogs with diabetes mellitus might be attributable in part to a decreased plasma leptin concentration.

In conclusion, the obese diabetic dogs had lower plasma leptin concentrations than the BCS-matched normal dogs. In dogs with diabetes mellitus, the plasma leptin concentration might be largely affected by factors other than adiposity.

\section{REFERENCES}

1. Al-Shoumer, K. A., Al-Asousi, A. A., Doi, S. A. and Vasanthy, B. A. 2008. Serum leptin and its relationship with metabolic variables in Arabs with type 2 diabetes mellitus. Ann. Saudi Med. 28: 367-370.

2. Considine, R. V. and Caro, J. F. 1997. Leptin and the regulation of body weight. Int. J. Biochem. Cell Biol. 29: 1255-1272.

3. Considine, R. V., Sinha, M. K., Heiman, M. L., Kriauciunas, A., Stephens, T. W., Nyce, M. R., Ohannesian, J. P., Marco, C. C., McKee, L. J., Bauer, T. L., et al. 1996. Serum immunoreactive-leptin concentrations in normal-weight and obese humans. N. Engl. J. Med. 334: 292-295.

4. Feldman, E. C. and Nelson, R. W. 2004. The endocrine pancreas. pp. 485-658. In: Canine and Feline Endocrinology and Reproduction 3rd ed., Saunders, Missouri.

5. Hathout, E. H., Sharkey, J., Racine, M., Ahn, D., Mace, J. W. and Saad, M. F. 1999. Changes in plasma leptin during the treatment of diabetic ketoacidosis. J. Clin. Endocrinol. Metab. 84: 4545-4548.

6. Ishioka, K., Hatai, H., Komabayashi, K., Soliman, M. M., Shibata, H., Honjoh, T., Kimura, K. and Saito, M. 2005. Diurnal variations of serum leptin in dogs: effects of fasting and refeeding. Vet. J. 169: 85-90.

7. Ishioka, K., Soliman, M. M., Sagawa, M., Nakadomo, F., Shi- bata, H., Honjoh, T., Hashimoto, A., Kitamura, H., Kimura, K. and Saito, M. 2002. Experimental and clinical studies on plasma leptin in obese dogs. J. Vet. Med. Sci. 64: 349-353.

8. Laferrere, B., Caixas, A., Fried, S. K., Bashore, C., Kim, J. and Pi-Sunyer, F. X. 2002. A pulse of insulin and dexamethasone stimulates serum leptin in fasting human subjects. Eur. $J$. Endocrinol. 146: 839-845.

9. Laflamme, D. P., Kuhlman, G., Lawler, D. F., Kealy, R. D. and Schmidt, D. A. 1994. Obesity management in dogs. Vet. Clin. Nutr. 1: 59-65.

10. Nishii, N., Takasu, M., Ohba, Y., Maeda, S., Kitoh, K., Ohtsuka, Y., Honjo, T., Saito, M. and Kitagawa, H. 2006. Effects of administration of glucocorticoids and feeding status on plasma leptin concentrations in dogs. Am. J. Vet. Res. 67: 266270.

11. Roden, M., Ludwig, C., Nowotny, P., Schneider, B., Clodi, M., Vierhapper, H., Roden, A. and Waldhausl, W. 2000. Relative hypoleptinemia in patients with type 1 and type 2 diabetes mellitus. Int. J. Obes. Relat. Metab. Disord. 24: 976-981.

12. Rojo-Martinez, G., Soriguer, F. J., Gonzalez-Romero, S., Tinahones, F., Moreno, F., de Adana, S. R., Garriga, M. J., Esteva, I., Garcia-Arnes, J., Gomez-Zumaquero, J. M. and GarciaAlmeida, J. M. 2000. Serum leptin and habitual fatty acid dietary intake in patients with type 1 diabetes mellitus. Eur. $J$. Endocrinol. 142: 263-268.

13. Sivitz, W. I., Wayson, S. M., Bayless, M. L., Larson, L. F., Sinkey, C., Bar, R. S. and Haynes, W. G. 2003. Leptin and body fat in type 2 diabetes and monodrug therapy. J. Clin. Endocrinol. Metab. 88: 1543-1553.

14. Verrotti, A., Basciani, F., Morgese, G. and Chiarelli, F. 1998. Leptin levels in non-obese and obese children and young adults with type 1 diabetes mellitus. Eur. J. Endocrinol. 139: 49-53.

15. Widjaja, A., Stratton, I. M., Horn, R., Holman, R. R., Turner, R. and Brabant, G. 1997. UKPDS 20: plasma leptin, obesity, and plasma insulin in type 2 diabetic subjects. J. Clin. Endocrinol. Metab. 82: 654-657.

16. Zhang, Y., Proenca, R., Maffei, M., Barone, M., Leopold, L. and Friedman, J. M. 1994. Positional cloning of the mouse obese gene and its human homologue. Nature 372: 425-432. 